\title{
Scale dependent continuum approaches for discontinuous assemblies: 'explicit' and 'implicit' non-local models
}

\author{
Meral Tuna ${ }^{\mathrm{a}}$, Patrizia Trovalusci ${ }^{\mathrm{b}, *}$ \\ ${ }^{a}$ Istanbul Technical University, Faculty of Mechanical Engineering, Istanbul, Turkey \\ ${ }^{b}$ Department of Structural and Geotechnical Engineering, Sapienza University of Rome, Via A. Gramsci, 53, 00197, Rome, Italy
}

\begin{abstract}
The aim of the present work is to investigate the mechanical behaviour of orthotropic masonry subjected to localized loads in the context of both 'implicit'/'weak' and 'explicit'/'strong' non-local continuum models. To look for possible correspondences and differences, the solutions of Cosserat (micropolar) and integral form of Eringen models, obtained by employing finite element method, are compared. The resulting displacement and stress fields highlight the diffusive character of micropolar model, and the capability of Eringen model in avoiding stress singularities.
\end{abstract}

Keywords: Cosserat, Eringen, Non-Local, Finite Element Method, Localised loads

\section{Introduction}

The gross mechanical behaviour of complex microstructured materials strongly depends on the heterogeneous and discontinuous nature of the underlying internal structure. ${ }_{35}$ 5 By lacking in internal scale parameters, the classical theory of elasticity seems incapable of capturing the size effects, while, direct discrete modelling techniques are computationally cumbersome for systems with many degree of freedoms [1, 2, 3]. To balance the accuracy with compu- ${ }_{40}$ 10 tational efficiency, it is favourable resorting to enhanced continuum theories $4,5,6,6,7,8,9$ which not only exploit the advantages of the field description at the coarse level, but also retain the memory of internal material organization. Depending on the procedures followed to incorpo- ${ }_{45}$ rate size effects, the non-classical theories are classified as 'implicit'/'weak' and 'explicit'/'strong', non-local models 4, 10, 11, 12. In the so-called implicit non-local models, also referred as multifield continua [12, the body is considered as a collection of deformable (or rigid) particles ${ }_{50}$ 20 that are endowed with additional degrees of freedom. As a result of additional descriptors introduced to represent the material microstructure, new strain/stress measures and balance equations are presented. On the other hand, explicit non-local models preserve the primal fields of clas- ${ }_{55}$ different operators or higher derivatives of the standard fields. Since implicit non-local models incorporates size effects only through newly introduced non-standard primal field, they possess a limited non-local character while as a ${ }_{60}$

\footnotetext{
* Corresponding author

Email addresses: tunamer@itu.edu.tr (Meral Tuna), patrizia.trovalusci@uniroma1.it (Patrizia Trovalusci)
}

explicit models have a strong non-local character. Among various implicit and explicit non-local models, the focus of the present work will be on the micropolar (Cosserat) theory [4, 7, 13, 14] and Eringen's nonlocal theory [9, 15]. Although both theories incorporate size effects and show spatial dispersion properties in wave propagation [6], application fields are quite different because of the distinct kinematic and static descriptors they possess. Micropolar model corresponds to an assembly of rigid particles which undergo displacement and rotations, independent of each other, and interact through forces and couples. It has been widely employed to describe many material cases and in particular to study block assemblies like granular rock, particle composites, masonry, etc. 16, 17, 18. It has been shown, moreover, that, due to the presence of the relative rotation between macro (local rigid) and micro rotation, that corresponds to the skew-symmetric part of strain, the micropolar continuum is particularly suitable to investigate the behaviour of anisotropic (specifically orthotropic) media 18, 19, 20, 21, 22. If micro rotations are constraint to follow the local rigid (macro) rotations, the theory becomes of Couple Stress, and by further adding statical constraint on couples the classical continuum is recovered (See for instance Appendix in 16]). On the other hand, Eringen's nonlocal theory of elasticity is concerned with the physics of materials bodies whose behaviour at a material point is influenced by the state of all points of the body 9 . Hence, the theory covers long-range interactions by relating stress at each point to the strain of entire domain through an attenuation-type kernel function containing information about non-locality. It has been widely employed to investigate the mechanical behaviour of nano or micro sized structures [23, 24, 25, 26, 27. As the ratio between nonlocal parameter and the external length of the 5 body approaches to zero, the size effects become negligible, 
and the model behaves as Cauchy continua.

Non-classical theories are used to study the various kinds of material problems [28, 29, but recently, a special attention is paid to comparison of various continuum 70 models which can shed light on the proper description of physical phenomena of the underlying discrete system [30, 31, 32, 33. To this end, possible correspondences and differences between 'implicit' Cosserat and 'explicit' Eringen theories are studied through a common problem of a 75 two-dimensional (2D) square masonry, fixed at the bottom edge and subjected to loads at the top edge (as in 34]). As in the previous exercise of the authors, focused on geometric singularity 33 , Eringen's theory is not here specifically used to model solids at atomistic scale, but rather the focus is on larger structures dominated by a mesoscale internal lenght, $l$, that does not significantly differ from the macroscopic length, $L\left(\frac{l}{L} \simeq 1\right)$. A block masonry wall has been considered, although the actual discrete structure of the materials is non-homogeneous, it has been described 85 as a homogeneous and orthotropic linear elastic equivalent continuum models, exploiting a coarse-graining procedure. This particular problem allows us to use a computationally efficient way to model class of structures, often encountered in historical masonry. Moreover, it allows us to serve the response of non-local continuum models in the presence of load singularities. The anisotropy, on the other hand, is incorporated to reflect response of masonry walls of practical interest; indeed, consideration of isotropy in continuum modelling corresponds to orthotetragonal ar95 rangements of bricks which has no practical importance. The numerical solution has been obtained using finite element formulations derived within the linearised kinematical framework. Devoting special attention to varying load footprint size and block/brick size, that is to varying the internal length and the level of non-locality, the response of Cosserat and Eringen continua is discussed focusing on resulting displacement and stress fields.

\section{Material and Methods}

In this section, continuum theories considered in the sudy are briefly explained, and the displacement-based finite element formulations are derived within the linearised kinematical framework. A Cartesian coordinate system $x y z$ is used with a suitable origin for parametrisation. The body under investigation is assumed as two-dimensional orthotropic, homogeneous medium with a uniform and symmetric thickening of $h$. The superscripts $E$ and $M$, that refer to Eringen's and micropolar model, respectively, are used to distinguish the parameters appearing in both theories, and possessing different interpretations. The sim-

ulations are performed by using an in-house Mathematica code considering two different discretisation and element formulations.

\subsection{Micropolar Theory}

Micropolar theory belongs to in a class of generalized continua, that using the definition [4, 10, 11, we call 'implicit' non-local model [12. The material particles that constitute the continuum are described in terms of both their positions and orientations ([4, 7, 13]). Hence, in the reduced $2 \mathrm{D}$ media, each particle possess two in-plane displacement components along $x$ and $y$ directions $\left(u_{x}, u_{y}\right)$, and one out-of-plane micro-rotation component along $\mathrm{z}$ direction $\left(\phi_{z}\right)$. In the linearised framework following kinematic relations are obtained:

$$
\varepsilon_{i j}^{M}=u_{i, j}+e_{i j k} \phi_{k}, \quad \chi_{k j}=\phi_{k, j}
$$

where, $\varepsilon_{i j}$ and $\chi_{k j}$ denote the components of strain and curvature tensor while $e_{i j k}$ being the usual third order perturbation tensor. With $\sigma_{i j}^{M}$ and $\mu_{k j}$ being the components of non-symmetric stress and couple stress tensors, following equilibrium equations are obtained in the absence of body forces and body couples:

$$
\sigma_{i j, j}^{M}=0, \quad \mu_{k j, j}-e_{i j k} \sigma_{i j}^{M}=0
$$

Considering linear elasticity, the stress-strain relation of an orthotropic micropolar continua can be represented as:

$$
\boldsymbol{\sigma}^{M}=\mathbf{D}_{\varepsilon}^{M} \varepsilon^{M}, \boldsymbol{\mu}=\mathbf{D}_{\chi} \chi
$$

with elasticity matrices being

$$
\begin{gathered}
\mathbf{D}_{\varepsilon}^{M}=\left[\begin{array}{cccc}
A_{1111} & A_{1122} & 0 & 0 \\
A_{2211} & A_{2222} & 0 & 0 \\
0 & 0 & A_{1212} & A_{1221} \\
0 & 0 & A_{2112} & A_{2121}
\end{array}\right] \\
\mathbf{D}_{\chi}=\left[\begin{array}{rr}
D_{11} & 0 \\
0 & D_{22}
\end{array}\right]
\end{gathered}
$$

By considering hyperelastic materials the major symmetries hold: $A_{i j h k}=A_{h k i j}, D_{i j}=D_{j i}(i, j, h, k=1,2)$. Note that, the elasticity constants appearing in $\mathbf{D}_{\varepsilon}^{M}$, do not retain memory of internal lengths, while these are incorporated in bending moduli $\mathbf{D}_{\chi}$, responsible for scale effects.

For FE modelling, the field variables within an element $e$ $\left(\mathbf{u}_{e}\right.$ and $\left.\phi_{e}\right)$ are approximated considering a natural coordinate system $\zeta, \eta$ and using related interpolation function matrices:

$$
\mathbf{u}_{e}=\mathbf{N}_{u}^{M} \tilde{\mathbf{u}}_{e}, \quad \phi_{e}=\mathbf{N}_{\phi} \tilde{\boldsymbol{\phi}}_{e}
$$

The over tilde symbol here is used to express the nodal values of field variables. Since optimum choice for micropolar element is nine-node Lagrange element [22, 34, 35] with micro-rotation DOFs attached only to the four corner nodes, the interpolation function matrices take the following form:

$$
\begin{aligned}
\mathbf{N}_{u}^{M}= & {\left[\begin{array}{lllll}
N_{u}^{1} & 0 & \ldots & N_{u}^{9} & 0 \\
0 & N_{u}^{1} & & 0 & N_{u}^{9}
\end{array}\right] } \\
& \mathbf{N}_{\phi}=\left[\begin{array}{lll}
N_{\phi}^{1} & \ldots & N_{\phi}^{4}
\end{array}\right]
\end{aligned}
$$


By using differential matrix operator, $\mathbf{L}^{M}$, permutation vector $\mathbf{M}$, and gradient operator, $\nabla$ :

$$
\begin{gathered}
\mathbf{L}^{M}=\left[\begin{array}{cccc}
\frac{\partial}{\partial x} & 0 & \frac{\partial}{\partial y} & 0 \\
0 & \frac{\partial}{\partial y} & 0 & \frac{\partial}{\partial x}
\end{array}\right]^{\mathrm{T}} \\
\mathbf{M}=\left[\begin{array}{llll}
0 & 0 & 1 & -1
\end{array}\right]^{\mathrm{T}} \\
\nabla=\left[\begin{array}{ll}
\frac{\partial}{\partial x} & \frac{\partial}{\partial y}
\end{array}\right]^{\mathrm{T}},
\end{gathered}
$$

the strain and curvature fields, ordered in vectors as

$$
\begin{gathered}
\varepsilon_{e}^{M}=\left[\begin{array}{cccc}
\varepsilon_{x x}^{M} & \varepsilon_{y y}^{M} & \varepsilon_{x y}^{M} & \varepsilon_{y x}^{M}
\end{array}\right]_{e}^{\mathrm{T}} \\
\chi_{e}=\left[\begin{array}{ll}
\chi_{z x} & \chi_{z y}
\end{array}\right]_{e}^{\mathrm{T}}
\end{gathered}
$$

are obtained:

$$
\begin{aligned}
& \varepsilon_{e}^{M}=\mathbf{L}^{M} \mathbf{u}_{e}+\mathbf{M} \phi_{e}=\mathbf{L}^{M} \mathbf{N}_{u}^{M} \tilde{\mathbf{u}}_{e}+\mathbf{M} \mathbf{N}_{\phi} \tilde{\phi}_{e} \\
& =\left[\begin{array}{ll}
\mathbf{L}^{M} \mathbf{N}_{u}^{M} & \mathbf{M} \mathbf{N}_{\varphi}
\end{array}\right]\left\{\begin{array}{c}
\tilde{\mathbf{u}}_{e} \\
\tilde{\boldsymbol{\phi}}_{e}
\end{array}\right\}=\mathbf{B}_{e \varepsilon}^{M} \mathbf{d}_{e}^{M}, \\
& \chi_{e}=\nabla \phi_{e}=\nabla\left(\mathbf{N}_{\phi} \tilde{\phi}_{e}\right) \\
& =\left[\begin{array}{cc}
0 & \nabla \mathbf{N}_{\phi}
\end{array}\right]\left\{\begin{array}{c}
\tilde{\mathbf{u}}_{e} \\
\tilde{\phi}_{e}
\end{array}\right\}=\mathbf{B}_{e \chi}^{M} \mathbf{d}_{e}^{M} \\
& \mathbf{d}_{e}^{M}=\left\{\begin{array}{llllllll}
\tilde{u}_{x}^{1} & \tilde{u}_{y}^{1} & \ldots & \tilde{u}_{x}^{9} & \tilde{u}_{y}^{9} & \tilde{\phi}_{z}^{1} & \ldots & \tilde{\phi}_{z}^{4}
\end{array}\right\}_{e}^{\mathrm{T}}
\end{aligned}
$$

where $\mathbf{B}_{e \varepsilon}^{M}$ and $\mathbf{B}_{e \chi}^{M}$ including derivation of interpolation functions called as strain-displacement matrices, while $\mathbf{d}_{e}^{M}$ refers to nodal displacement vector. Note that the superscripts appeared in Eqs. (6) and $\sqrt{9} 3$ refer to node numbers, and the derivation operations given in Eq. (7) are performed using chain rule and the inverse of Jacobian matrix. For the uniform mesh configuration considered in the present study, the Jacobian matrix is same for all square elements with an edge length of $l_{e}$ :

$$
\mathbf{J}=\frac{l_{e}}{2}\left[\begin{array}{ll}
1 & 0 \\
0 & 1
\end{array}\right]
$$

Inherently, for an element $e$ the constitutive equations given in Eq. (3) are transformed to

$$
\boldsymbol{\sigma}_{e}^{M}=\mathbf{D}_{e \varepsilon}^{M} \mathbf{B}_{e \varepsilon}^{M} \mathbf{d}_{e}^{M}, \quad \boldsymbol{\mu}_{e}=\mathbf{D}_{e \chi}^{M} \mathbf{B}_{e \chi}^{M} \mathbf{d}_{e}^{M}
$$

with

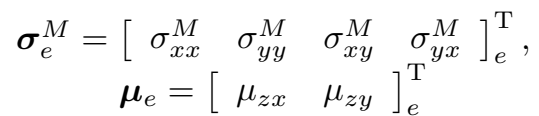

As the last step, the minimum of total potential energy principle is employed to obtain the formulation of an element $m$ with $\mathbf{f}_{m}^{M}$ and $\mathbf{k}_{m}^{M}$ being the element force vector and element stiffness matrix:

$$
\mathbf{k}_{m}^{M}=\underbrace{\left(\begin{array}{c}
h \\
h \int_{-1}^{1} \int_{-1}^{1}\left(\mathbf{B}_{m \varepsilon}^{M}\right)^{\mathrm{T}} \mathbf{D}_{m \varepsilon}^{M} \mathbf{B}_{m \varepsilon}^{M} \mathbf{d}_{m}^{M} \operatorname{det}\left|\mathbf{J}_{m}\right| \mathrm{d} \zeta \mathrm{d} \eta \\
+\int_{-1}^{1} \int_{-1}^{1}\left(\mathbf{B}_{m \chi}^{M}\right)^{\mathrm{T}} \mathbf{D}_{m \chi}^{M} \mathbf{B}_{m \chi}^{M} \operatorname{det}\left|\mathbf{J}_{m}\right| \mathrm{d} \zeta \mathrm{d} \eta
\end{array}\right)}_{\mathbf{k}_{m \varepsilon}^{M}+\mathbf{k}_{m \chi}^{M}}
$$

Eventually, by performing proper assemblage operations, the linear equation system is achieved:

$$
\mathbf{K}^{M} \mathbf{d}^{M}=\mathbf{f}^{M}
$$

where $\mathbf{K}^{M}, \mathbf{d}^{M}$ and $\mathbf{f}^{M}$ refer to global stiffness matrix, global nodal displacement vector, and global external force vector of a $2 \mathrm{D}$ micropolar FE model. For integrations, a standard numerical integration technique, Gauss quadrature rule, is employed with either two or three Gauss sampling points depending on the order of the elements.

\subsection{Integral form of Eringen's nonlocal theory}

Eringen's theory is considered as an 'explicit' non-local model 4, 10, 11, where stress at each point is related to strain of entire domain through an attenuation-type kernel function [9, 15. In this theory, primal fields of classical elasticity are conversed and following kinematical relations are obtained within the linearised framework:

$$
\varepsilon_{i j}^{E}=\frac{1}{2}\left(u_{i, j}+u_{j, i}\right)
$$

where $\varepsilon_{i j}^{E}$ denotes components of symmetric strain tensor. In the absence of body forces, following equilibrium equation similar to classical elasticity theory is carried out:

$$
\sigma_{i j, j}^{E}=0
$$

with $\sigma_{i j}^{E}$ being the components of symmetric stress tensor. Considering linear elasticity, the stress-strain relation of an orthotropic Eringen media is represented as follows

$$
\boldsymbol{\sigma}^{E}=\iint \tau(r, \kappa) \mathbf{D}^{E} \boldsymbol{\varepsilon}^{E}(\bar{x}, \bar{y}) d \bar{A}
$$

where $\tau(r, \kappa)$ refers to kernel function, $\bar{A}$ corresponds to surface area of the $2 \mathrm{D}$ domain, $r$ denotes the Euclidean distance between corresponding point $\mathbf{x}(x, y)$ and its neighbouring points $\overline{\mathbf{x}}(\bar{x}, \bar{y})$ (i.e. $r=|\mathbf{x}-\overline{\mathbf{x}}|$ ) and $\kappa$ is the non-local parameter including information about internal length, and where:

$$
\mathbf{D}^{E}=\left[\begin{array}{ccc}
\hat{A}_{1111} & \hat{A}_{1122} & 0 \\
\hat{A}_{2211} & \hat{A}_{2222} & 0 \\
0 & 0 & \hat{A}_{1212}
\end{array}\right]
$$

Note that, in the present study, the orthotropic material behaviour is included only through bulk properties, while 
kernel function is assumed as isotropic similar to exist135 that the anisotropic material behaviour through not only elastic stiffness properties, but also length scale parameters is topic of an ongoing project. In this study, hyperelastic materials are considered in such a way that: $\hat{A}_{i j h k}=\hat{A}_{h k i j},(i, j, h, k=1,2)$.

For FE modelling, the domain is discretised with $2 \mathrm{D}$ elements, and the displacement field within an linear element $e$ is approximated by using interpolation function matrix and corresponding nodal displacement vector:

$$
\begin{aligned}
& \mathbf{u}_{e}=\mathbf{N}_{u}^{E} \mathbf{d}_{e}^{E} \\
& \mathbf{N}_{u}^{E}=\left[\begin{array}{lllllll}
N_{u}^{1} & 0 & \ldots & N_{u}^{4} & 0 \\
0 & N_{u}^{1} & \cdots & 0 & N_{u}^{4}
\end{array}\right] \\
& \mathbf{d}_{e}^{E}=\left\{\begin{array}{llllllll}
\tilde{u}_{x}^{1} & \tilde{u}_{y}^{1} & \tilde{u}_{x}^{2} & \tilde{u}_{y}^{2} & \tilde{u}_{x}^{3} & \tilde{u}_{y}^{3} & \tilde{u}_{x}^{4} & \tilde{u}_{y}^{4}
\end{array}\right\}_{e}{ }^{\mathrm{T}}
\end{aligned}
$$

According strain field within an element $e$ (i.e. $\varepsilon_{e}^{E}=$ $\left[\begin{array}{ccc}\varepsilon_{x x}^{E} & \varepsilon_{y y}^{E} & 2 \varepsilon_{x y}^{E}\end{array}\right]_{e}^{T}$ ) is then calculated as follows by using the differential matrix operator, $\mathbf{L}^{E}$ :

$$
\begin{aligned}
& \varepsilon_{e}^{E}=\mathbf{L}^{E} \mathbf{N}_{u}^{E} \mathbf{d}_{e}^{E}=\mathbf{B}_{e}^{E} \mathbf{d}_{e}^{E} \\
& \mathbf{L}^{E}=\left[\begin{array}{ccc}
\frac{\partial}{\partial x} & 0 & \frac{\partial}{\partial y} \\
0 & \frac{\partial}{\partial y} & \frac{\partial}{\partial x}
\end{array}\right]^{T}
\end{aligned}
$$

As already mentioned, the derivations with respect to physical coordinate systems given in Eq. 20$]_{2}$ are performed by the chain rule, and employing the inverse of Jacobian matrix of element. Consequently, for an exponential type kernel function, the constitutive equation given in Eq. (17) is transformed to following for an element $e$ :

$$
\sigma_{e}^{E}=\sum_{i=1}^{N_{\text {total }}} \int_{-1}^{1} \int_{-1}^{1} \frac{\mathrm{e}^{-\frac{r}{\kappa}}}{2 \pi \kappa^{2}} \mathbf{D}_{i}^{E} \overline{\mathbf{B}}_{i}^{E} \mathbf{d}_{i}^{E} \operatorname{det}\left|\overline{\mathbf{J}}_{i}\right| \mathrm{d} \bar{\zeta} \mathrm{d} \bar{\eta}
$$

where stress tensor is described as:

$$
\sigma_{e}^{E}=\left[\begin{array}{ccc}
\sigma_{x x}^{E} & \sigma_{y y}^{E} & \sigma_{x y}^{E}
\end{array}\right]_{e}^{T}
$$

The subscript $i$ in Eq. 21) refers to elements that fall within the influence zone of the element $e$, hence $N_{\text {total }_{e}}$ being the total number of related neighbour elements of $e$. Their numbers increase with non-locality and disrupts the banded character of global stiffness matrix. Also note that ${ }_{160}$ the over bar denotes that the related matrix is written in terms of $\bar{\zeta}, \bar{\eta}$ due to long range interactions in constitutive equation.

Based on the minimum of total potential energy principle, the formulation of $m$ th element of Eringen nonlocal ${ }_{165}$ model is derived as below:

$$
\mathbf{f}_{m}^{E}=2 \mathbf{k}_{m m}^{E} \mathbf{d}_{m}^{E}+\sum_{n=1, n \neq m}^{N_{\text {total }_{m}}}\left(\mathbf{k}_{m n}^{E} \mathbf{d}_{n}^{E}+\left(\mathbf{k}_{n m}^{E}\right)^{T} \mathbf{d}_{n}^{E}\right)
$$

with

$$
\begin{aligned}
\mathbf{k}_{m n}^{E} & =\frac{h}{2} \int_{-1}^{1} \int_{-1}^{1} \int_{-1}^{1} \int_{-1}^{1} \frac{\mathrm{e}^{-\frac{r}{\kappa}}}{2 \pi \kappa^{2}} \mathbf{L}_{m n} \mathrm{~d} \bar{\zeta} \mathrm{d} \bar{\eta} \mathrm{d} \zeta \mathrm{d} \eta \\
\mathbf{L}_{m n} & =\left(\mathbf{B}_{m}^{E}\right)^{T} \mathbf{D}_{n}^{E} \overline{\mathbf{B}}_{n}^{E} \operatorname{det}\left|\overline{\mathbf{J}}_{n}\right| \operatorname{det}\left|\mathbf{J}_{m}\right|
\end{aligned}
$$

and

$$
r=\sqrt{(\bar{\zeta}-\zeta)^{2}+(\bar{\eta}-\eta)^{2}}
$$

Note that for a domain with homogeneous material properties, as in the present work, following simplification can be made:

$$
\mathbf{k}_{m n}^{E}=\left(\mathbf{k}_{n m}^{E}\right)^{T}
$$

Finally, a linear equation system is obtained similar to Cosserat model by performing proper assemblage operations with $\mathbf{K}^{E}, \mathbf{d}^{E}$ and $\mathbf{f}^{E}$ being the global stiffness matrix, global nodal displacement vector, and global external force vector of a 2D Eringen FE model.

$$
\mathbf{K}^{E} \mathbf{d}^{E}=\mathbf{f}^{E}
$$

To calculate the integration in Eq. 24 with minimum possible error, the number of Gauss sampling points should be carefully adjusted depending on the ratio between nonlocal parameter and element length $\left(\kappa / l_{e}\right)$. As this ratio decreases, more Gauss points are required to catch the trend of exponential function in integration.

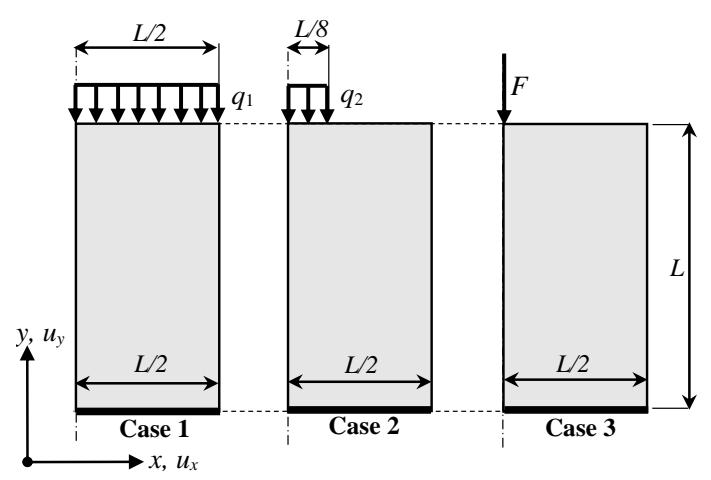

Figure 1: Geometric sketch of the problem under investigation

\section{Numerical Simulations}

The present study aimed at comparing the two different non-local models; namely 'explicit'/'strong' Eringen model and 'implicit'/'weak' Cosserat model in the presence of localised loads and accountable size effects. To this end, a square wall of length $L=4 \mathrm{~m}$ and thickness $h=1 \mathrm{~m}$, fixed at the bottom edge and subjected to several loads at the top edge, is investigated. As illustrated through half wall geometries at Fig. 11, the footprint size and intensity of loads are arranged to keep the resultant force, $F$, constant, such that; $q_{1}=2.5 \mathrm{MPa}, q_{2}=10.0 \mathrm{MPa}$, $F=10 \mathrm{MN}$. It must be noted that, to avoid any numerical problems (hourglassing) that may encounter for increasing $\kappa / l_{e}$ ratios, concentrated load is modelled as a distributed load on a very small region $(<L / 50)$ for Eringen's model. Furthermore, to examine the scale effects, the structures are investigated considering two different internal lengths (brick/block sizes), resulting in altered non-locality. The corresponding continuum material properties of Cauchy, Cosserat and Eringen models are listed in Table 1. 
Table 1: Constitutive material parameters used in calculations Cauchy

\begin{tabular}{ll}
\hline$\hat{A}_{1111}=3.75 \times 10^{4} \mathrm{MPa}$ \\
$\hat{A}_{2222}=1.50 \times 10^{4} \mathrm{MPa}$ \\
$\hat{A}_{1212}=1.875 \times 10^{4} \mathrm{MPa}$ \\
\hline \multicolumn{2}{c}{ Cosserat } \\
\hline \multicolumn{3}{c}{ parameter set 1: } & \multicolumn{1}{c}{$l_{1} / L=0.1$} \\
$A_{1111}=3.75 \times 10^{4} \mathrm{MPa}$ & $\left(D_{11}\right)_{1}=450.0 \mathrm{MN}$ \\
$A_{2222}=1.50 \times 10^{4} \mathrm{MPa}$ & $\left(D_{22}\right)_{1}=150.0 \mathrm{MN}$ \\
$A_{1212}=0.75 \times 10^{4} \mathrm{MPa}$ & parameter set 2: \\
$A_{2121}=3.00 \times 10^{4} \mathrm{MPa}$ & $l_{2} / L=0.25$ \\
& $\left(D_{11}\right)_{2}=2812.5 \mathrm{MN}$ \\
& $\left(D_{22}\right)_{2}=937.5 \mathrm{MN}$ \\
\hline \multicolumn{2}{c}{$\mathrm{Eringen}$} \\
\hline$\hat{A}_{1111}=3.75 \times 10^{4} \mathrm{MPa}$ & parameter set 1: \\
$\hat{A}_{2222}=1.50 \times 10^{4} \mathrm{MPa}$ & $\kappa_{1}=0.012 \mathrm{~m}$ \\
$\hat{A}_{1212}=1.875 \times 10^{4} \mathrm{MPa}$ & parameter set 2: \\
& $l_{2} / L=0.25$ \\
& $\kappa_{2}=0.03 \mathrm{~m}$
\end{tabular}

For Cosserat and Cauchy continua, the equivalent material constants are evaluated using the coarse graining approach given in [38, while the non-local parameter in Eringen model is selected based on the variation of displacement field between Cauchy and Cosserat models in such a way that, the vertical displacement at the middle of the top edge is almost same for both models in Case 2. This can be achieved simply by trial and error or using an evolutionary algorithm previously employed [27. The main reason attention is focused on Case 2 in parametrization is because the size effects are pronounced for both non-classical models (unlike Case 1) and it does not possess singularity (unlike Case 3 ). The ratio between first and second non-local parameter is then arranged to be in accordance with the change in the block size.

The analysis are repeated for two different mesh configurations illustrated at Fig. 2 2 Note that we assume non-dilatant material for which: $A_{1122}=A_{2211}=A_{1221}=$ $A_{2112}=0$ as well as $\hat{A}_{1122}=\hat{A}_{2211}=0$.

In the following, comments about each load case will be235 briefly given with focusing on resulting vertical displacement and stress fields demonstrated in Figs. 3 and 4 for Mesh 1. It is important to mention that, the alteration of results with mesh configuration is only evident in the proximity of localised loads, and still lead to same contour ${ }_{240}$ plots for considered legends.

According to results, following inferences can be made. For 'implicit' non-local models, size effects are not implied under uniform distributed load (Case 1) which results in a homogeneous deformation state, while the devi-245 increases with non-locality is originated from long range

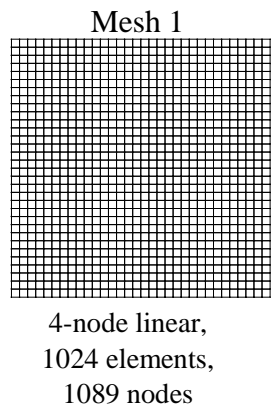

(a)

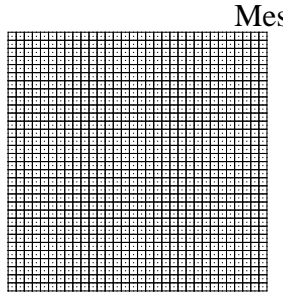

9-node Lagrange, 1024 elements 4225 nodes

(b)

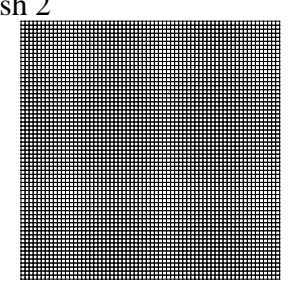

4-node linear, 4096 elements, 4225 nodes

(c)

Figure 2: Discretisation of the domain (a) coarse mesh configuration, (b), (c) fine mesh configuration

interactions and missing neighbour relations. For other cases yielding inhomogeneous deformations (Case 2 and 3), Cosserat and Eringen models act in an opposite manner non-locality, Eringen model becomes more flexible while Cosserat model becomes more rigid. As a consequence, Cosserat is able to better distribute the load depending on the level of non-locality, more in the presence of larger 5 tered

To show the effect of non-locality on both non-classical models, the variation of vertical stress along vertical axis $y$ is illustrated at Fig. 5 for Cases 2 and 3. Although the advantageous character of Eringen's model in providing finite stresses with problems having singularities are reported many times, an interesting point draws one's attention. As a consequence of missing neighbour interactions around boundaries, the corresponding regions become more flexible, and results in an abrupt increase of stress. Although for Case 1 and 2, it results in slightly higher values inside the domain close to boundary (see Fig. 5(a), for concentrated load case, the incorporation of long range interactions compensates that looseness and leads to finite stress field (see Fig. 5(b)). This behaviour is in agreement with that reported by Eringen [9, where the displacement field of local theory is used for calculation of stress. Hence, the reason why the maximum stress in the present study is estimated higher than Eringen [9] is due to no assumption made on displacement fields.

In terms of computational efficiency, Cauchy and Cosserat model is absolutely superior over Eringen, because of two reasons. In Eringen model, due to incorporation of long range interactions, the strain field of all elements located in the influence zone of corresponding element should be considered during forming the element stiffness matrix. This necessity intensively extends the calculations, and disrupts the banded character of global stiffness matrix. Secondly, due to incorporation of kernel function in element formulation, the number of Gauss sampling points should be drastically increased with increasing non-locality or decreasing element length in order 

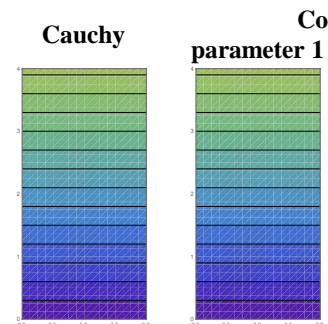

Cosserat
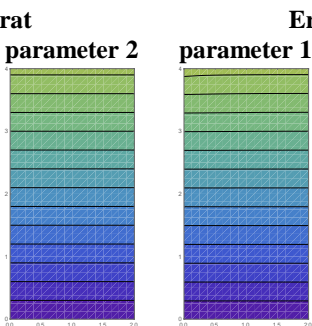

ringen
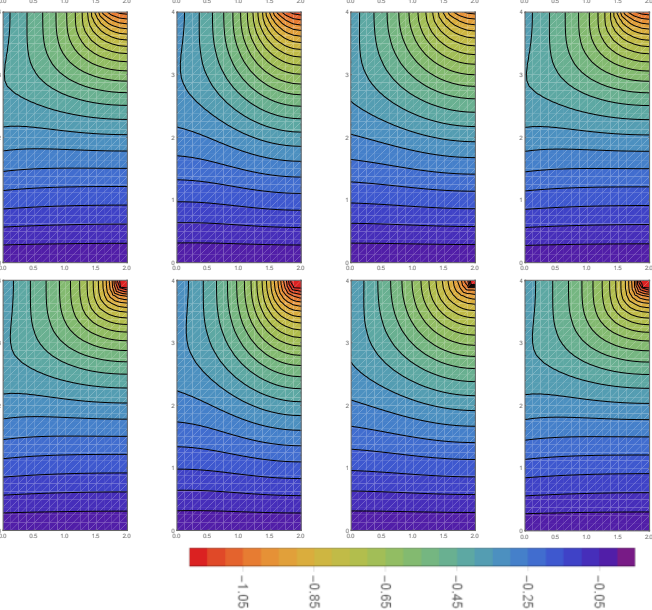

Figure 3: Vertical displacement fields $\left(u_{y} \times 10^{3}\right)$ for Case 1 (1st row), Case 2 (2nd row), and Case 3 (3rd row)

to calculate the integrals with minimum possible error.

\section{Final Remarks}

Providing that a constitutive characterization obtainable via multiscale procedures, as the ones in [18, 19] or via optimization approaches [27, both the 'explicit' and ${ }^{280}$ 'implicit' type non-local theories can be effectively used for representing the mechanical behaviour of complex materials, as composites or masonry-like materials, by retaining memory of the material microstructure avoiding the resort to computationally burdensome discrete modelling. The result of the comparison between Cosserat 'implicitly' and Eringen 'explicitly' non-local models highlights that,285 the former should be preferred not only for the high computational efficiency and ease of implementation but also because it shows better performance in reproducing the actual behaviour of masonry wall, due to the presence of 290 the relative rotation related to the skew-symmetric part of the strain 22, 39, 20. However, the advantage of Eringen model in providing finite stresses in case of load singularities 9] should not be ignored. Further investigation ${ }^{295}$ may be conducted on the wave propogation and dispersion problems, in which the size effects are important, by
Cauchy
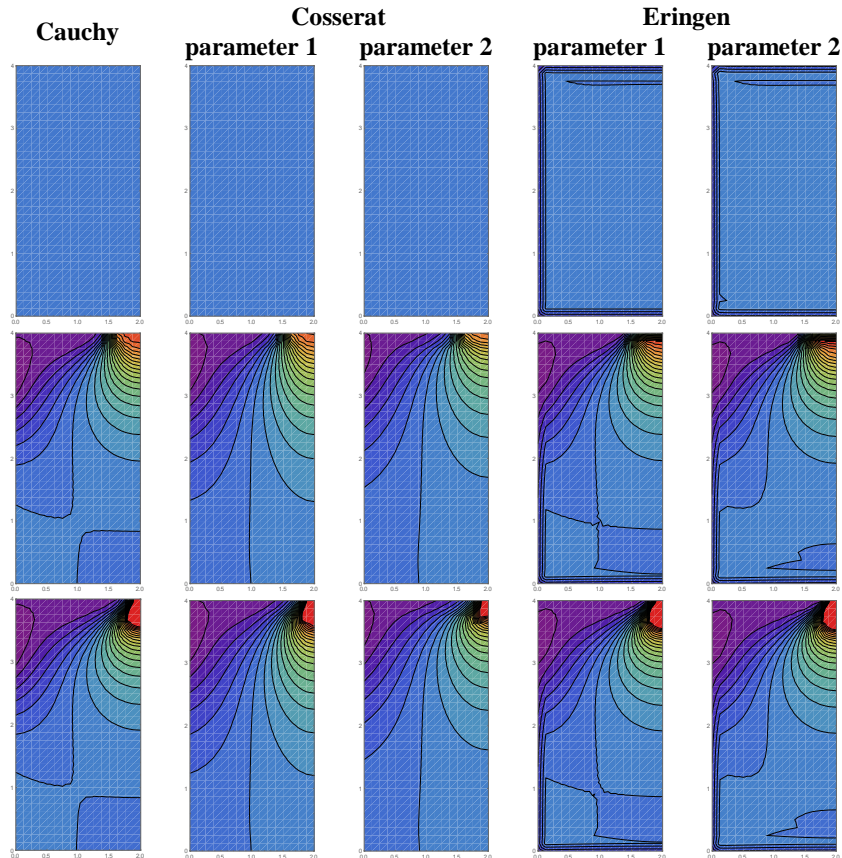

ringen

parameter 2
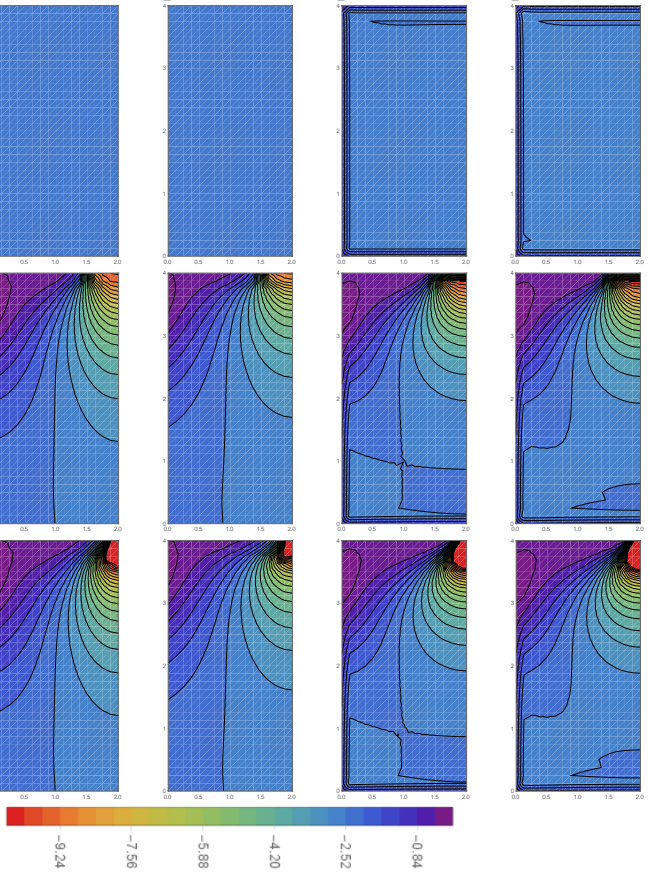

Figure 4: Vertical stress fields $\left(\sigma_{y y}\right)(\mathrm{MPa})$ for Case 1 (1st row), Case 2 (2nd row), and Case 3 (3rd row)

\section{Acknowledgements}

This work was done when Meral Tuna was a Visiting Researcher at DISG, Sapienza University of Rome, with financial support of Italian Ministry of University and Research PRIN 2015, project 2015JW9NJT (Grant No. B86J16002300001, ID: 10.13039/501100003407) "Materials with microstructure: multiscale models for the derivation non-local continua of 'implicit/weak' and explicit/strong"' which is gratefully acknowledged.

Professor Patrizia Trovalusci acknowledges the support of MIUR/Sapienza Progetti Medi 2017 (Grant No. B83C17001440005), and MIUR/PRIN 2017 (Grant No. B86J16002300001).

\section{References}

[1] D. Rapaport, The Art of Molecular Dynamics Simulation, 2nd Edition, Cambridge University Press, 2004.

[2] D. Yang, Y. Sheng, J. Ye, Y. Tan, Discrete element modeling of the microbond test of fiber reinforced composite, Computational Materials Science 49 (2) (2010) 253 - 259.

[3] E. Reccia, L. Leonetti, P. Trovalusci, A. Cecchi, A multiscale/multidomain model for the failure analysis of masonry walls: A validation with a combined fem/dem approach, International Journal for Multiscale Computational Engineering 16 (2018) 325-343.

[4] A. Eringen, Microcontinuum Field Theory, Springer, 1999.

[5] R. D. Mindlin, Micro-structure in linear elasticity, Archive for Rational Mechanics and Analysis 16 (1) (1964) 51-78.

[6] I. A. Kunin, The theory of elastic media with microstructure and the theory of dislocations, in: E. Kröner (Ed.), Mechanics 

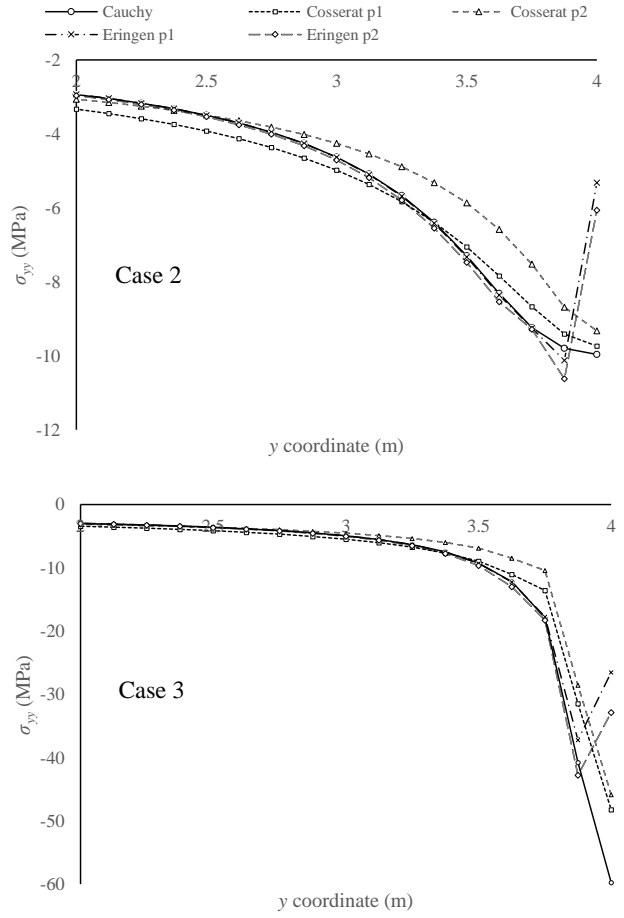

Figure 5: $\sigma_{y y}$ along the vertical axis $y$

of Generalized Continua, Springer Berlin Heidelberg, 1968, pp. 321-329.

[7] G. Capriz, Continua with Microstructure, Springer Tracts in Natural Philosophy, Springer-Verlag, 1989.

[8] M. Gurtin, Configurational Forces as Basis Concept of Continuum Physics, Springer-Verlag, 1999.

[9] A. Eringen, Nonlocal Continuum Field Theories, SpringerVerlag, 2002.

[10] I. Kunin, On foundations of the theory of elastic media with $_{380}$ microstructure, International Journal of Engineering Science $22(8)(1984) 969-978$.

[11] G. Maugin, Material Inhomogeneities in Elasticity, Applied Mathematics, Taylor \& Francis, 1993.

[12] P. Trovalusci, Molecular approaches for multifield continua: origins and current developments, in: T. Sadowski, P. Trovalusci (Eds.), Multiscale Modeling of Complex Materials: Phenomenological, Theoretical and Computational Aspects, Springer Vienna, 2014, pp. 211-278.

[13] W. Nowacki, Theory of Asymmetric Elasticity, Elsevier Science ${ }_{390}$ \& Technology, 1986.

[14] H. Altenbach, V. A. Eremeyev, Cosserat media, in: H. Altenbach, V. A. Eremeyev (Eds.), Generalized Continua from the Theory to Engineering Applications, Springer Vienna, Vienna, 2013, pp. 65-130.

[15] A. Eringen, D. Edelen, On nonlocal elasticity, International Journal of Engineering Science 10 (3) (1972) 233 - 248.

[16] R. Masiani, P. Trovalusci, Cosserat and cauchy materials as continuum models of brick masonry, Meccanica 31 (4) (1996) 421-432.

[17] S. Forest, K. Sab, Cosserat overall modeling of heterogeneous materials, Mechanics Research Communications 25 (4) (1998) $449-454$.

[18] P. Trovalusci, R. Masiani, Material symmetries of micropolar continua equivalent to lattices, International Journal of Solids ${ }_{405}$ and Structures 36 (14) (1999) $2091-2108$.

[19] P. Trovalusci, A. Pau, Derivation of microstructured continua from lattice systems via principle of virtual works: the case of masonry-like materials as micropolar, second gradient and classical continua, Acta Mechanica 225 (1) (2014) 157-177.

[20] V. A. Eremeyev, W. Pietraszkiewicz, Material symmetry group and constitutive equations of micropolar anisotropic elastic solids, Mathematics and Mechanics of Solids 21 (2) (2016) 210221.

[21] M. Godio, I. Stefanou, K. Sab, J. Sulem, S. Sakji, A limit analysis approach based on cosserat continuum for the evaluation of the in-plane strength of discrete media: Application to masonry, European Journal of Mechanics - A/Solids 66 (2017) 168 -192 .

[22] N. Fantuzzi, L. Lorenzo, T. Patrizia, T. Francesco, Some novel numerical applications of cosserat continua, International Journal of Computational Methods 15 (06) (2018) 1850054.

[23] C. Polizotto, Nonlocal elasticity and related variational principles, International Journal of Solid and Structures 38 (2001) $7359-7380$.

[24] A. A. Pisano, A. Sofi, P. Fuschi, Finite element solutions for nonhomogeneous nonlocal elastic problems, Mechanics Research Communications 36 (7) (2009) $755-761$.

[25] H. M. Numanoglu, B. Akgoz, O. Civalek, On dynamic analysis of nanorods, International Journal of Engineering Science 130 (2018) 33-50.

[26] M. Tuna, M. Kirca, P. Trovalusci, Deformation of atomic models and their equivalent continuum counterparts using eringen's two-phase local/nonlocal model, Mechanics Research Communications 97 (2019) $26-32$.

[27] M. Tuna, M. Kirca, Unification of eringen's nonlocal parameter through an optimization-based approach, Mechanics of Advanced Materials and Structures (2019).

[28] B. Akgoz, O. Civalek, Effects of thermal and shear deformation on vibration response of functionally graded thick composite microbeams, Composites Part B: Engineering 129 (2017) 77 87.

[29] C. Demir, O. Civalek, A new nonlocal fem via hermitian cubic shape functions for thermal vibration of nano beams surrounded by an elastic matrix, Composite Structures 168 (2017) $872-884$.

[30] N. Challamel, C. Wang, I. Elishakoff, Nonlocal or gradient elasticity macroscopic models: A question of concentrated or distributed microstructure, Mechanics Research Communications 71 (2016) $25-31$.

[31] V. A. Eremeyev, G. Rosi, S. Naili, Comparison of anti-plane surface waves in strain-gradient materials and materials with surface stresses, Mathematics and Mechanics of Solids 24 (8) (2019) 2526-2535.

[32] V. Eremeyev, B. Sharma, Anti-plane surface waves in media with surface structure: Discrete vs. continuum model, International Journal of Engineering Science 143 (2019) 33 - 38.

[33] M. Tuna, L. Leonetti, P. Trovalusci, M. Kirca, 'Explicit' and 'implicit' non-local continuous descriptions for a circular plate with an inclusion in tensions, Submitted to Meccanica.

[34] L. Leonetti, N. Fantuzzi, P. Trovalusci, F. Tornabene, Scale effects in orthotropic composite assemblies as micropolar continua: A comparison between weak- and strong-form finite element solutions, Materials 12 (5) (2019).

[35] N. Fantuzzi, P. Trovalusci, S. Dharasura, Mechanical behavior of anisotropic composite materials as micropolar continua, Frontiers in Materials 6 (2019) 59.

[36] Y. Sun, Z. Zhou, Stress field near the crack tip in nonlocal anisotropic elasticity, European Journal of Mechanics - A/Solids 23 (2) (2004) $259-269$.

[37] Z. Zhou, B. Wang, Investigation of the interaction of two collinear cracks in anisotropic elasticity materials by means of the nonlocal theory, International Journal of Engineering Science 43 (13) (2005) $1107-1120$.

[38] P. Trovalusci, R. Masiani, Non-linear micropolar and classical continua for anisotropic discontinuous materials, International Journal of Solids and Structures 40 (2003) 1281-1297.

[39] A. Pau, P. Trovalusci, Block masonry as equivalent micropolar continua: the role of relative rotations, Acta Mechanica 223 (7) (2012) 1455-1471. 